\title{
Melamine-based Covalent Organic Polymers (MCOPs) as Lipase Nanocarrier for Recyclable Esters Hydrolysis and Transesterification
} \author{
Zhihao Wang ${ }^{1 \dagger}$, Ying $\mathrm{Chen}^{1 \dagger}$, Jiahe Zhao', Guoliang Gao ${ }^{2}$, Worawan Panpipat ${ }^{3}$,
} Ling-Zhi Cheong ${ }^{1 *}$, and Cai Shen ${ }^{2}$

\footnotetext{
${ }^{1}$ Department of Food Science and Engineering, College of Food and Pharmaceutical Sciences, Ningbo University, Ningbo 315832, CHINA

${ }^{2}$ Institute of Materials Technology \& Engineering, Chinese Academy of Sciences, 1219 Zhongguan Road, Ningbo 315201, CHINA

${ }^{3}$ Food Technology and Innovation Research Center of Excellence, Department of Agro-Industry, School of Agricultural Technology, Walailak University, Thasala, Nakhon Si Thammarat 80161, THAILAND

${ }^{\dagger}$ These authors contributed equally to the work
}

\begin{abstract}
Present study has successfully synthesized melamine-based covalent organic polymers (MCOPs) and applied it as lipase carrier for recyclable esters hydrolysis and transesterification. The synthesized MCOPs are composed of dense nanosheet structures having a thickness of $3.5 \mathrm{~nm}$. Three immobilization methods namely physical adsorption, cross-linking and carrier activation were employed to prepare the MCOPs-immobilized CRL. Cross-linked MCOPs-immobilized CRL (41.30 mg protein/g MCOPs) and carrier activated MCOPs-immobilized CRL (33.20 mg protein/g MCOPs) had higher enzyme loading as compared to physical absorb MCOPs-immobilized CRL (29.30 mg protein/g MCOPs). Nevertheless, physical absorb MCOPs-immobilized CRL demonstrated the highest esters hydrolysis (49.85 $\mathrm{U})$ and transesterification (1.04 U) activities. Despite having the highest enzymatic activity, physical absorb MCOPs-immobilized CRL were not able to maintain its catalytic stability with more than $30 \%$ decreased in enzymatic activity during consecutive hydrolysis and transesterification activities. Meanwhile, cross-linked MCOPs-immobilized CRL demonstrated highest catalytic stability with highest enzymatic activities at the end of consecutive reactions. All the MCOPs-immobilized CRL can be easily recovered and reused through centrifugation with more than $85 \%$ of recovery rate.
\end{abstract}

Key words: melamine-based covalent organic polymers, Candida rugosa lipase, immobilization, hydrolysis, transesterification

\section{Introduction}

Green chemistry refers to production of chemical compounds based on green processes, emphasizes the use of renewable resources and environmentally friendly process$\mathrm{es}^{1}{ }^{1}$. Enzymes which can be obtained from renewable sources and capable of catalyzing various chemical reactions under mild conditions are an important part of green chemistry. Lipases (triacylglycerol ester hydrolases, EC 3.1.1.3), which catalyze hydrolysis and synthesis of longchain acylglycerols and many other esters in aqueous/ organic biphasic media, are one of the most widely used biocatalysts in organic chemistry ${ }^{2}$. They are able to preserve their catalytic activity in organic solvents, biphasic systems and micellar solutions ${ }^{3)}$. Candida rugosa lipase
(CRL, $6.46 \times 6.31 \times 4.77 \mathrm{~nm}$ ) is one of the most commonly used lipases and has been widely used for biosynthesis and biotransformations such as resolution of racemic acids, high enantioselective esterification and selective hydrolysis of saturated fatty acids. CRL has great application potentials in chemical and biological industries ${ }^{4-7)}$. However, high cost, marginal stability in harsh reaction conditions and difficulties in recovery and reusability has limit its industrial application ${ }^{8)}$.

Enzyme immobilization refers to confinement or localization of enzymes within a defined space which allows the enzymes to be separated physically from substrate and product for reuse ${ }^{9)}$. Enzyme immobilization is also able to increase enzyme catalytic stability in harsh environment ${ }^{10}$.

\footnotetext{
*Correspondence to: Ling-Zhi Cheong, Department of Food Science and Engineering, College of Food and Pharmaceutical Sciences, Ningbo University, Ningbo 315832, CHINA

E-mail: cheonglingzhi@nbu.edu.cn

Accepted February 23, 2020 (received for review February 5, 2020)

Journal of Oleo Science ISSN 1345-8957 print / ISSN 1347-3352 online

http://www.jstage.jst.go.jp/browse/jos/ http://mc.manusriptcentral.com/jjocs
} 
Lipases had been immobilized on various inorganic (Hydrotalcite, GO/ZnO composite, metals, controlled pore metal oxides) and organic (agarose, chitin, collagen, nylon, cellulose) carriers using either non-covalent physical adsorption, encapsulation, covalent bonding and cross-linking methods ${ }^{11-14)}$. Some of these carriers, however, may cause enzyme conformational transition and decrease mass transfer resulting in low biocatalytic activity ${ }^{15}$.

Nanosheets materials have recently been used for enzyme immobilization ${ }^{16-18)}$. Covalently-linked porous organic polymers (COPs), is a novel nanosheets material constructed through covalent bonds. It possess unique properties including high porosity, high surface area, low density, good thermal and solvent stability ${ }^{19)}$. COPs have been applied in gas storage/separation, catalysis, metal adsoprtion, concentration of phenolic antioxidants and sensing ${ }^{19,20)}$. Melamine (MA), $66 \% \mathrm{~N}$ by mass, is a cheap and abundant triazine monomer used extensively in plastic, medicinal, decorative, and paper industries. One of the unique properties of MA include its molecular recognition ability through donation and acceptance of hydrogen bonds, metal chelation, and $\pi-\pi$ interactions. Recently, MA has been used as monomers for synthesis of MA-based COPs (MCOPs) with high porosity, special surface morphology and amorphous nanostructures which are applied for adsorption of heavy metal ions ${ }^{19)}$. The aforementioned unique characteristics of MCOPs has deemed it a promising novel candidate for enzyme immobilization. Nevertheless, limited studies have been conducted to date to investigate the use of this unique materials for enzyme immobilization.

Present study aimed to synthesize MCOPs and investigate its application as carrier for CRL (Fig. 1). CRL was immobilized on the MCOPs using three methods namely physical adsorption, cross-linking and carrier activation. Catalytic activity of the MCOPs-immobilized CRLs was evaluated in terms of esters hydrolysis and transesterification activities. In addition, recovery and reusability of the MCOPs-immobilized CRLs were also investigated through consecutive hydrolysis and transesterification reactions. Findings from present study will guide development of a metal-free organic polymer materials for lipase immobilization.

\section{Experimental Procedures}

\subsection{Materials}

Candida rugosa lipase (CRL) was obtained from Amano, JAPAN. $p$-Nitrophenyl palmitate ( $p$-NPP) and Bicinchoninic acid assay (BCA) were purchased from Sigma Aldrich.
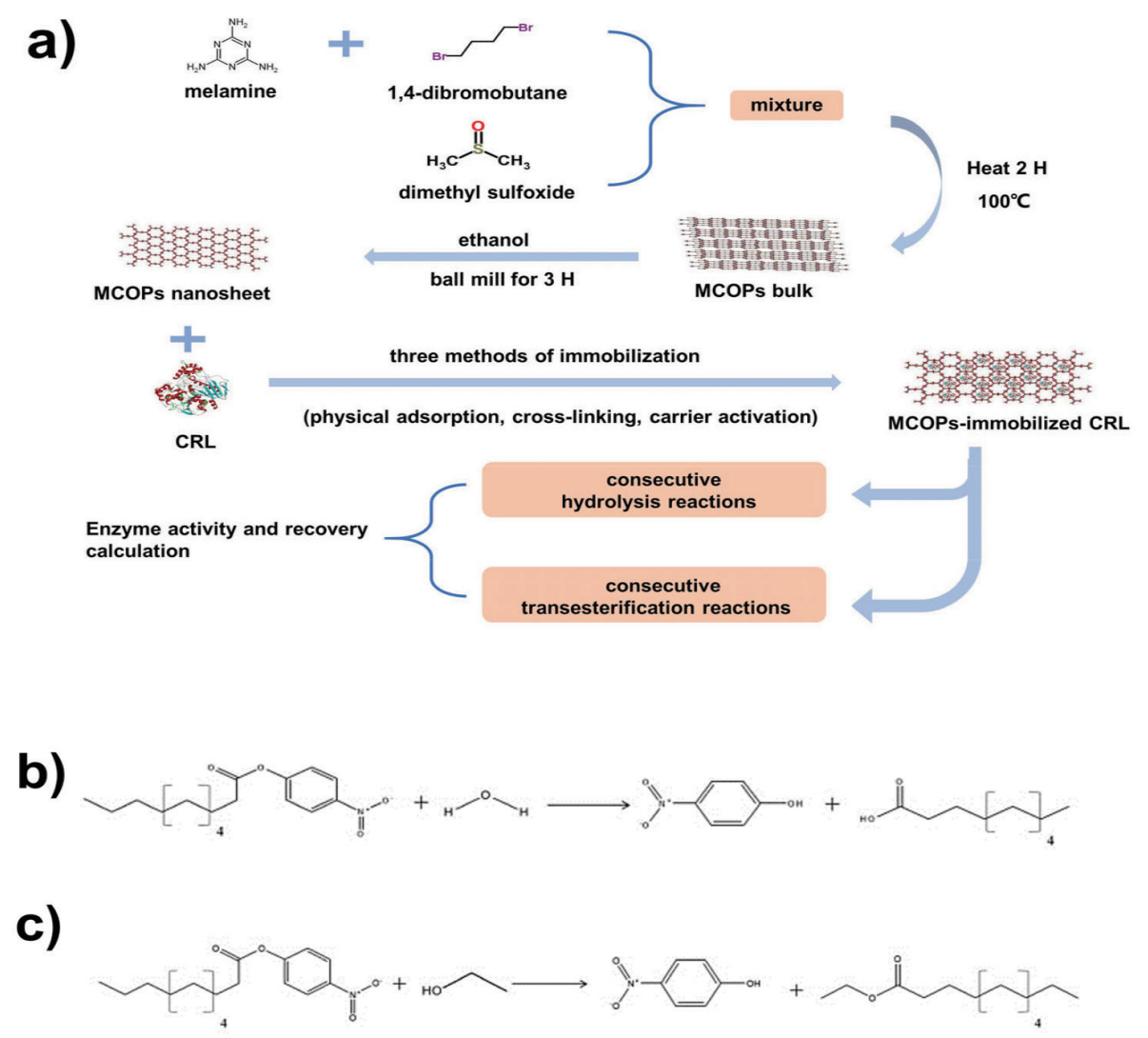

Fig. 1 a) Experimental flow diagram. b) Hydrolysis and c) transesterification reactions catalyzed by MCOPs-immobilized CRL. 
Sodium bicarbonate $\left(\mathrm{Na}_{2} \mathrm{CO}_{3}\right)$, phosphate buffer $(\mathrm{pH} 7.0)$, ethanol, $n$-hexane, melamine, dimethyl sulfoxide (DMSO), 1,4-dibromobutane and acetone were obtained from Aladdin Chemicals.

\subsection{Apparatus}

Fourier-transform infrared spectroscopy (Nicolet 6700, Thermo-fisher, USA), Bruker's Dimension icon Atomic Force Microscope, Hitachi Scanning Electron Microscope (SEM, Hitachi TM3030).

\subsection{Measurements}

\subsubsection{Synthesis of MCOPs}

Synthetic paths in references ${ }^{21)}$. Firstly, $0.939 \mathrm{~g}$ of melamine was added to a beaker containing $50 \mathrm{~mL}$ of dimethyl sulfoxide. The mixture was magnetically stirred for $10 \mathrm{~min}$ to form a homogeneous solution. Following that, $1.36 \mathrm{~mL}$ of 1,4-dibromobutane was added to the mixture and stirred $30 \mathrm{~min}$. The resultant mixture was then heated at $100^{\circ} \mathrm{C}$ for $2 \mathrm{~h}$. Acetone was then added to the mixture to obtain a yellowish product. The yellowish product was purified by centrifugation and washed 6 times with a mixture of acetone and methanol (volume ratio of 1:1) to remove residual DMSO. The purified product was dissolved in absolute ethanol and ballmilled (600 r/min) for $3 \mathrm{~h}$ to obtain MCOPs. Finally, the obtained MCOPs was vacuum dried at $80^{\circ} \mathrm{C}$ for $10 \mathrm{~h}$.

\subsubsection{Physical characterization of the MCOPs}

MCOPs was characterized using Fourier-transform infrared analysis (FTIR), atomic force microscope (AFM) and scanning electron microscopy (SEM). FTIR analysis was conducted using a Fourier-transform infrared spectroscopy. The incident light was oriented at the Brewster angle of silicon of $74^{\circ}$, and spectra between 650 and $4000 \mathrm{~cm}^{-1}$ were acquired over $100 \mathrm{scans}$ with a resolution of $8 \mathrm{~cm}^{-1}$. AFM analysis was conducted using Bruker's Dimension icon under ambient condition with a silicon nitride cantilever (SNL-10) using a measured spring constant of $0.4 \mathrm{~N} / \mathrm{m}$. All AFM images were acquired in ScanAsyst mode with an image resolution of $256 \times 256$ pixels. Structural morphology of the MCOPs was observed and imaged using a focused ion beam scanning electron microscope (Carl Zeiss) with a working potential of $3 \mathrm{kV}$ under magnifications ranging from $7.35 \mathrm{~K}$ to $44.87 \mathrm{~K}$.

\subsubsection{Immobilization of CRL on MCOPs}

CRL was immobilized on MCOPs using three different methods namely physical adsorption, cross-linking and carrier activation according to previously published method with slight modifications ${ }^{22)}$.

\subsubsection{Physical adsorption method}

Twenty five milligram of MCOPs was mixed with $2.5 \mathrm{~mL}$ of CRL solution[10 mg CRL/mL of phosphate buffer $(\mathrm{pH}$ 7.0) ]. The resulting mixture was shake in incubator $(280$ rpm) for $8 \mathrm{~h}$ at $40^{\circ} \mathrm{C}$. At the end of the immobilization process, the mixture was centrifuged $(6000 \mathrm{rpm} / \mathrm{min})$ for 10 min and precipitates were collected as MCOPs-immobilized CRL (PA).

2.3.3.2 Cross-linking method

Twenty five milligram of MCOPs was mixed with $2.5 \mathrm{~mL}$ of CRL solution[10 mg CRL/mL of phosphate buffer ( $\mathrm{pH}$ 7.0) ]. The resulting mixture was shake in incubator $(280$ rpm) for $8 \mathrm{~h}$ at $40^{\circ} \mathrm{C}$. Following that, the mixture was centrifuged (6000 rpm/min) for $10 \mathrm{~min}$ and precipitates were collected and immersed in $5 \mathrm{~mL}$ of $25 \%$ glutaraldehyde ( 5 $\min$.

2.3.3.3 Carrier activation method

MCOPs was activated by immersing in $5 \mathrm{~mL}$ of $25 \%$ glutaraldehyde solution for 5 min. Following that, MCOPs were separated by centrifugation at $6000 \mathrm{rpm} / \mathrm{min}$ for 10 min. Activated MCOPs was mixed with $2.5 \mathrm{~mL}$ CRL solution [10 mg CRL/mL phosphate buffer $(\mathrm{pH} 7.0)]$ and shake in incubator $(280 \mathrm{rpm} / \mathrm{min})$ for $8 \mathrm{~h}$ at $40^{\circ} \mathrm{C}$. At the end of the immobilization process, the mixture was centrifuged (6000 rpm/min) for $10 \mathrm{~min}$ and precipitates were collected as MCOPs-immobilized CRL (CA).

All the MCOPs-immobilized CRL was washed with phosphate buffer to remove excess of unbound CRL. The immobilized BCL was vacuum dried and stored at $4^{\circ} \mathrm{C}$. Enzyme loading was quantified using BCA assay with bovine serum albumin (BSA) as standard and calculated using the following equation:

Enzyme loading $(\mathrm{mg} / \mathrm{g}$ particles $)=\frac{\mathrm{m}-\mathrm{cv}}{\mathrm{w}} \times 100 \%$

Whereby $\mathrm{m}(\mathrm{mg})$ refers to CRL concentration in phosphate buffer; $\mathrm{c}(\mathrm{mg} / \mathrm{mL})$ and $\mathrm{v}(\mathrm{mL})$ refer to CRL concentration and volume of the supernatant, respectively; $\mathrm{w}(\mathrm{g})$ refers to the weight of MCOPs. Determination of enzyme loading was conducted in triplicate.

2.3.4 Catalytic activity of the MCOPs-immobilized CRL

Esters hydrolysis activity was determined according to a previously reported method with slight modifications ${ }^{23)}$. Four milliliters of ethanol containing $p$-NPP $(15 \mathrm{mM})$ and phosphate buffer solution ( $4 \mathrm{~mL}$ of $50 \mathrm{mM}$, pH 7.5) were mixed in a screw-capped $15 \mathrm{~mL}$ vial. Twenty five milligram of MCOPs-immobilized CRL was then added to initiate the hydrolysis reaction. The mixture was incubated at $37^{\circ} \mathrm{C}$ in incubator shaker (300 rpm) for $15 \mathrm{~min}$. Reaction was terminated by adding $4 \mathrm{~mL}$ of $0.5 \mathrm{M} \mathrm{Na}_{2} \mathrm{CO}_{3}$ followed by centrifugation at $6000 \mathrm{rpm}$ for $10 \mathrm{~min}$. The supernatant $(0.5 \mathrm{~mL})$ was diluted 10-fold with deionized water and 4-Nitrophenol ( $p$-NP) liberated was extracted by aqueous alkaline phase, and detected at $410 \mathrm{~nm}$ against a blank without enzyme using UV-vis spectrophotometer. The amount of liberated $p$-NP was determined according to standard curve prepared by varying amounts of $p$-NP. Enzyme activity (U) was defined as amounts of $p$-NP liberated per min by per gram of MCOPs-immobilized CRL. All reactions were performed in triplicate. 
Esters of transesterification activity was conducted using method by Teng et al. ${ }^{24)}$. MCOPs-immobilized CRL (25 mg) was added to screw-capped vials containing $10 \mathrm{mM} p$-NP and ethanol $(300 \mu \mathrm{L})$ and $n$-hexane $(5 \mathrm{~mL})$. Transesterification was performed at $40^{\circ} \mathrm{C}$ in a shaking incubator $(200$ $\mathrm{rpm}$ ) for $45 \mathrm{~min}$. At the end of the reaction, $\mathrm{Na}_{2} \mathrm{CO}_{3}$ solution $(1 \mathrm{~mL})$ was added to terminate the reaction. The reaction mixture was centrifuged at $6000 \mathrm{rpm}$ for $10 \mathrm{~min}$ and lower aqueous phase was diluted 5 times and 4-Nitrophenol $(p-\mathrm{NP})$ liberated was quantified at $410 \mathrm{~nm}$. Enzyme activity $(\mathrm{U})$ was defined as amounts of $p$-NP liberated per min by per gram of MCOPs-immobilized CRL. All reactions were performed in triplicate.

2.3.5 Catalytic stability of the MCOPs-immobilized CRL

Catalytic stability of the MCOPs-immobilized CRL was evaluated by consecutive hydrolysis and transesterification reactions. Following each reaction, MCOPs-immobilized CRL was washed three times with phosphate buffer $(5 \mathrm{~mL})$ to remove residual unreacted $p$-NPP from the immobilized enzyme. MCOPs-immobilized CRL was dried under nitrogen stream to constant weight and the recovered immobilized CRL was weighed. Enzyme recovery rate is defined as the ratio of the mass of MCOPs recovered after each reaction to the mass of MCOPs before the start of the reaction. The recovered immobilized CRL was then subjected to subsequent esters hydrolysis or transesterification reactions. Enzyme activity for each cycle of reaction was determined according to aforementioned procedures.

\section{Results and Discussion}

\subsection{Physical characterization of the MCOPs}

MCOPs were successfully synthesized and characterized. Figure $2 a$ shows the FTIR spectra of melamine and the synthesized MCOPs. FTIR spectra of the melamine demonstrated the absorption bands near $3469,3419 \mathrm{~cm}^{-1}$ correspond to the anti-symmetric stretching vibration of $\mathrm{NH}_{2}$ and the absorption bands near $3332,3129 \mathrm{~cm}^{-1}$ correspond to the bending vibration of $\mathrm{NH}_{2}{ }^{25,26)}$. Following complete reaction with 1,4 dibromoethane, the reaction product (MCOPs) did not contain any absorption peaks at 3469 , $3419,3332,3129 \mathrm{~cm}^{-1}$ indicating complete reaction of the melamine. Instead, the reaction product (MCOPs) demonstrated new peak at 2917 and $2836 \mathrm{~cm}^{-1}$ which was attributed to bending of the $\mathrm{CH}_{2}$. Formation of these new peaks indicated that 1,4-dibromoethane has successfully incorporated hydrocarbon groups into the MCOPs. In addition, MCOPs also demonstrated absorption peak at 1650 and $1550 \mathrm{~cm}^{-1}$ which are due to bending vibration of $\mathrm{NH}_{2}$ and stretching vibration of $\mathrm{C}=\mathrm{N}$, respectively. A peak at 814 $\mathrm{cm}^{-1}$ which represents the bending vibration of triazine can also be observed. Specific details of the FTIR spectra and functional groups of melamine and MCOPs are shown in Table 1.

Figure 2b-c shows morphology of the MCOPs as imaged using SEM and AFM. MCOPs is mainly composed of dense nanosheet structures which are stacked in layers. AFM analysis shows the MCOPs nanosheet has a thickness of 3.5 nm (Fig. 2d).

\subsection{Immobilization of the CRL on MCOPs}

Three different approaches namely physical adsorption,
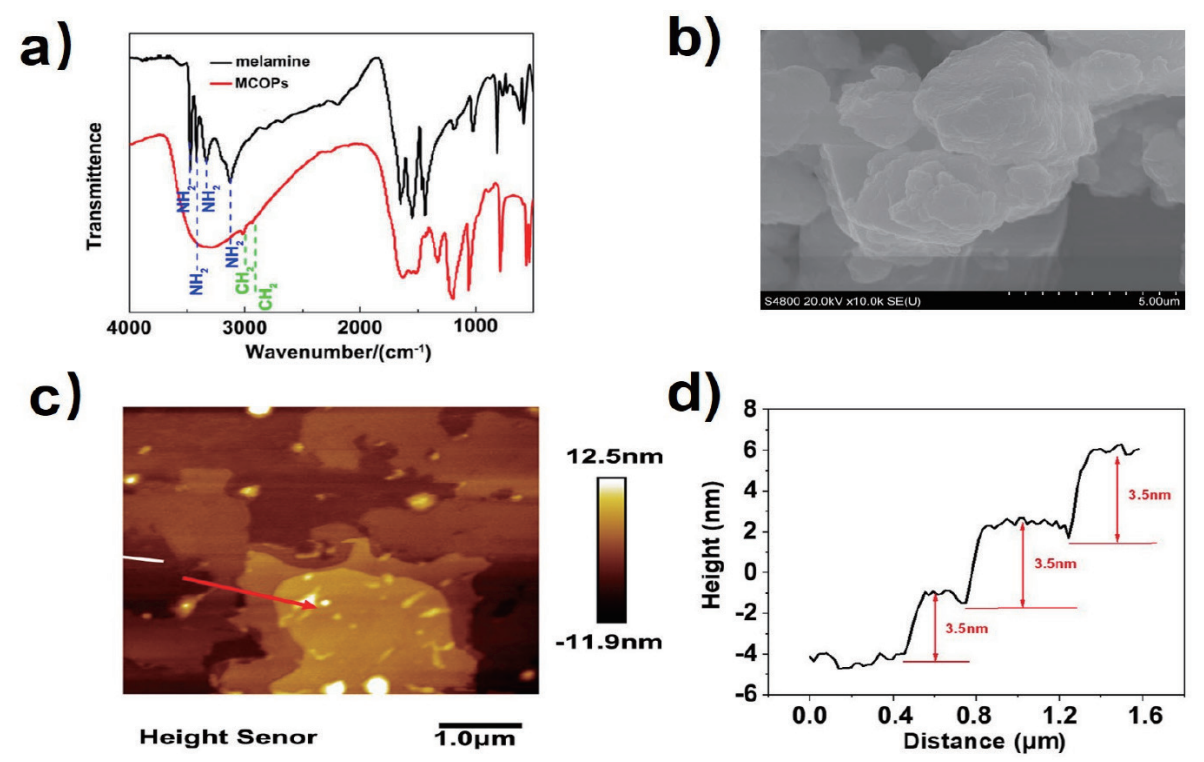

Fig. 2 a) FTIR spectra of the melamine and MCOPs. b) SEM image of the MCOPs. c) AFM image of MCOPs nanosheet and d) Height profile of the MCOPs nanosheet. 
cross-linking and carrier activation was used to immobilized the CRL on MCOPs. Among the three methods, crosslinking method demonstrated the highest CRL loading rate of $41.30 \mathrm{mg}$ protein/g MCOPs, followed by carrier activation and physical adsorption methods which have CRL loading rate of $33.20 \mathrm{mg}$ protein/g MCOPs and $29.30 \mathrm{mg}$ protein/g MCOPs, respectively. Higher loading rate of the cross-linking and carrier activation methods can be attributed to the use of glutaraldehyde which introduces reactive functional group forming Schiff base to bind with the CRL enzyme $^{27,28)}$.

\subsection{Catalytic activity of the MCOPs-immobilized CRL}

Figure 3 shows the ester hydrolysis and transesterification activities of the MCOPs-immobilized CRL obtained through different immobilization methods. In comparison to free CRL which has a hydrolysis activity of $93 \mathrm{U}$, all the MCOPs immobilized-CRL showed decreased in hydrolysis activity (physical adsoprtion: $44.81 \mathrm{U}$, cross linking: $40.25 \mathrm{U}$ and carrier activation: $49.85 \mathrm{U}$ ). Similar trend can be observed for esters transesterification activity. Immobilization resulted in a decrease in transesterification activity from $1.2 \mathrm{U}$ (free CRL) to $1.04 \mathrm{U}$ (physical adsoprtion), $0.878 \mathrm{U}$ (cross-linking) and $0.729 \mathrm{U}$ (carrier activation), respectively. Decrease in enzyme activity can be attributed to changes in enzyme comformation following binding with the MCOPs carrier. In addition, immobilization may have decrease the mass transfer rate between the immobilized CRL and $p$-NPP substrate ${ }^{7)}$. Interestingly, reduction in hydrolytic activity of the MCOPs-immobilized CRL is more pronounced as compared to the transesterification activity of lipases. As transesterification is performed in an organic solvent system, low reduction of transesterification activity of the MCOPs-immobilized CRL indicated that MCOPs-immobilized CRL may demonstrate good solvent stability.

\subsection{Catalytic stability of the MCOPs-immobilized CRL}

Figure 4 shows the catalytic stability of the MCOPs-immobilized CRL in consecutive hydrolysis and transesterification reactions.

An interesting phenomenon was observed during the consecutive hydrolysis reactions. Regardless of the immo-

Table 1 FTIR spectra of melamine and MCOPs.

\begin{tabular}{ccc}
\hline Group & Vibration & $\begin{array}{c}\text { Wavelength } \\
\left(\mathrm{cm}^{-1}\right)\end{array}$ \\
\hline $\mathrm{N}-\mathrm{H}$ & Stretching & 3469 \\
$\mathrm{~N}-\mathrm{H}$ & Stretching & 3419 \\
$\mathrm{~N}-\mathrm{H}$ & Bending & 3332 \\
$\mathrm{~N}-\mathrm{H}$ & Bending & 3129 \\
$\mathrm{CH}_{2}$ & Bending & 2917 \\
$\mathrm{CH}$ & Bending & 2836 \\
$\mathrm{~N}-\mathrm{H}$ & Bending & 1650 \\
$\mathrm{C}=\mathrm{N}$ & Stretching & 1550 \\
$\mathrm{~N}-\mathrm{H}$ & Ring out of plane deformation & 1024 \\
triazine & Bending & 814 \\
\hline
\end{tabular}

a)

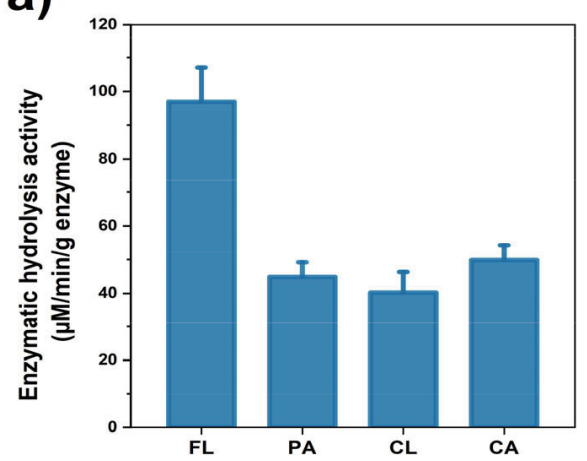

b)

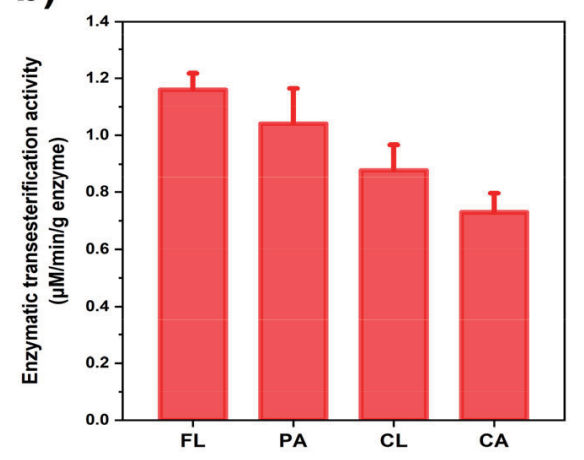

Fig. 3 a) Esters hydrolysis and b) transesterification activities of free lipase (FL), physical adsorb (PA), cross-linked (CL), and carrier activated (CA) MCOPs-immobilized CRL. 
bilization methods, all the MCOPs-immobilized CRL showed a two-fold increased in terms of hydrolytic activity in the second cycle during consecutive hydrolysis reaction. It is possible that the MCOPs-immobilized CRL has their essential water layer surronding the enzyme molecules stripped off during the immobilization process resulting in lower hydrolytic activity as compared to free CRL. The MCOPs-immobilized CRL then has regained essential water layer to maintain their optimal hydrolytic activity from the first hydrolysis cycle ${ }^{7}$.

Among the three immobilization methods, MCOPs-immobilized CRL obtained through physical adsorption method has the highest enzyme activity during the first few reactions with a hydrolytic activity of $10.93 \mathrm{U}$ during the second cycle. Nevertheless, there is a $31 \%$ dropped in hydrolytic activity to $7.54 \mathrm{U}$ during the sixth cycle (Fig. $4 a$ ). We postulated that the weak binding force of physical adsorption method has led to lipases leakage and eventually decreased catalytic activity during the recycle test.

MCOPs-immobilized CRL obtained through cross-linking method also demonstrated siimlar trend with high hydrolytic activity during first few cycles of the hydrolysis reactions. Nevertheless, cross-linked MCOPs-immobilized CRL demonstrated higher catalytic stability as compared to physical adsorb MCOPs-immobilized CRL. Cross-linked MCOPs-immobilized CRL recorded a 19.05\% decreased in hydrolytic activity ( $8.33 \mathrm{U}$ ) during the sixth cycle. In the seventh cycle, cross linked MCOPs-immobilized CRL has the highest hydrolytic activity of $5.98 \mathrm{U}$ followed by carrier activated MCOPs-immobilized CRL (3.76 U) and physical adsorb MCOPs-immobilized CRL (3.49 U). In short, physical adsorb MCOPs-immobilized CRL has the highest hydrolytic activity but its catalytic stability is inferior to crosslinked MCOPs-immobilized CRL.

In terms of transesterification activities, regardless of immobilization methods, there were no significant differences in transesterification activity during the consecutive reactions (Fig. 4b). Similar to hydrolysis reaction, physical adsorb MCOPs-immobilized CRL demonstrated the highest transesterification activity but cross-linked MCOPs-immobilized CRL demonstrated better catalytic stability. After seven consecutive transesterification reactions, crosslinked MCOPs-immobilized CRL was able to maintain $82.5 \%$ of its transesterification activity. Meanwhile, physical absorbed and carrier activated MCOPs-immobilized CRL were only able to maintain $68.7 \%$ and $58.26 \%$ of its initial activities, respectively.

It is worth noting that both the cross-linked and carrier activated MCOPs-immobilized CRL has lower catalytic activity as compared to physical adsorb MCOPs-immobilized CRL. This may due to changes in the CRL conformation as glutaraldehyde has been reported to reduce substrate affinity ${ }^{29)}$. a)

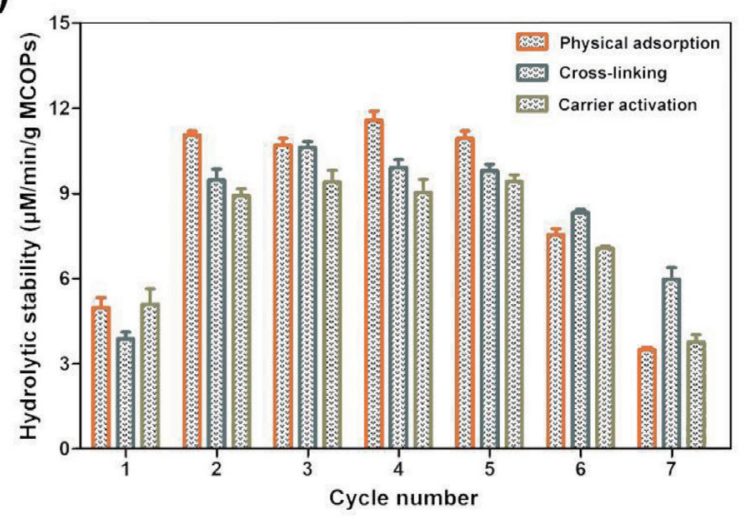

b)

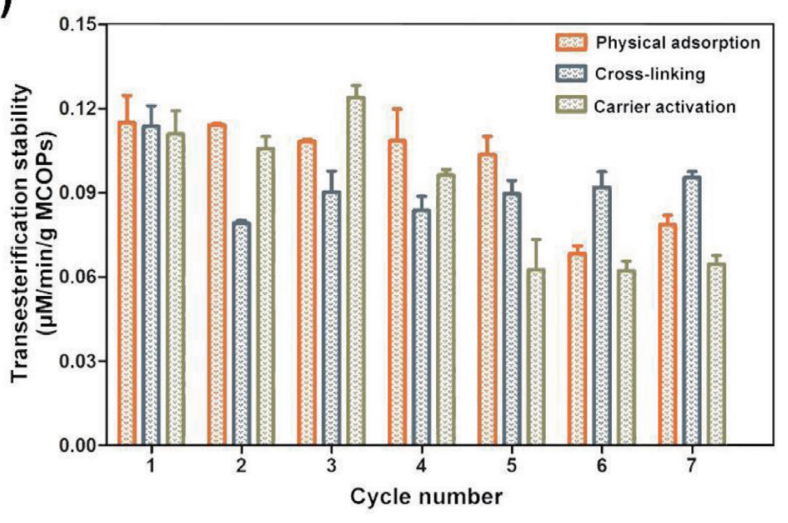

Fig. 4 a) Hydrolytic and b) transesterification activities of the MCOPs-immobilized CRL obtained using physical adsorption, cross-linking and carrier activation methods.

\subsection{Recovery of the MCOPs-immobilized CRL}

Figure 5 shows the recovery of MCOPs-immobilized CRL in consecutive hydrolysis and transesterification reactions. Regardless of immobilization methods, all the MCOPs-immobilized CRL can be easily recovered through centrifugation process during consecutive hydrolysis and transesterification processes. More than $85 \%$ of MCOPs-immobilized CRL can be recovered during the repeated experiments. Both aqueous and organic solvents did not affect the recovery of the MCOPs-immobilized CRL indicating good recyclability of the immobilized CRL.

\section{Conclusion}

MCOPs is successfully synthesized and applied as carrier for CRL for esters hydrolysis and transesterifications. MCOPs-immobilized CRL produced through cross-linking and carrier activation methods had higher enzyme loading as compared to MCOPs-immobilized CRL produced through physical adsorption method. Nevertheless, physi- 
a)

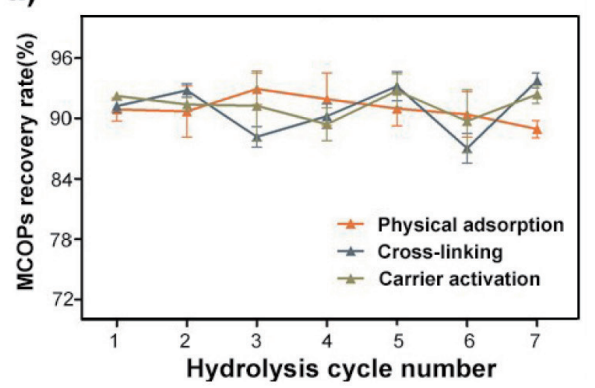

b)

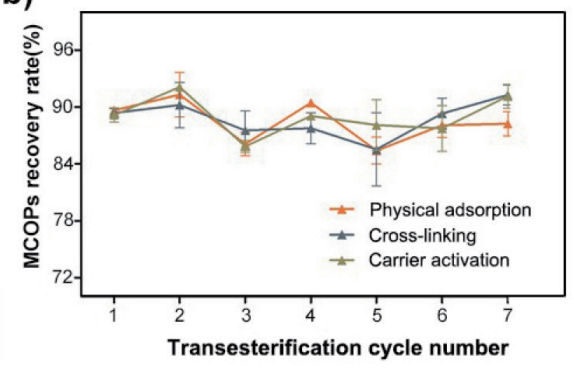

Fig. 5 Recovery of the MCOPs-immobilized CRL during consecutive a) hydrolysis and b) transesterification reactions.

cal absorb MCOPs-immobilized CRL demonstrated the highest esters hydrolysis and transesterification activities. Despite having highest enzymatic activity, physical absorb MCOPs-immobilized CRL were not able to maintain its catalytic stability with more than $30 \%$ decreased in enzymatic activity during consecutive hydrolysis and transesterification activities. Among all the immobilized CRL, cross-linked MCOPs-immobilized CRL demonstrated the highest catalytic stability with the highest enzymatic activities at the end of the consecutive reactions. All the MCOPs-immobilized CRL can be easily recovered and reused with more than $85 \%$ of recovery rate. Findings from present study will guide development of MCOPs for used as a novel carrier for lipase which can be used in wide range of applications involving esters hydrolysis and transesterification reactions.

\section{Acknowledgment}

This work was sponsored by National Natural Science Foundation of China (21706137) and Ningbo University (421401560). Ling-Zhi Cheong acknowledged funding support from Ningbo "3315” Talent Program.

\section{References}

1) Anastas, P.; Eghbali, N. Green chemistry: principles and practice. Chem. Soc. Rev. 39, 301-312 (2010).

2) Cheong, L.-Z.; Wei, Y.; Wang, H.; Wang, Z.; Su, X.; Shen, C. Facile fabrication of a stable and recyclable lipase@amine-functionalized ZIF-8 nanoparticles for esters hydrolysis and transesterification. J. Nanopart. Res. 19, 280 (2017).

3) Villeneuve, P.; Muderhwa, J.M.; Graille, J.; Haas, M.J. Customizing lipases for biocatalysis: a survey of chemical, physical and molecular biological approaches. $J$. Mol. Catal. B 9, 113-148(2000).

4) Sánchez, A.; RíO, J.L.D.; Valero, F.; Lafuente, J.; Faus, I.; Solà, C. Continuous enantioselective esterification of trans-2-phenyl-1-cyclohexanol using a new Candida rugosa lipase in a packed bed bioreactor. J. Biotechnol. 84, 1-12(2000).

5) Dong-Lin, W.; Ahindra, N.; Guan-Chun, L.; Jei-Fu, S. Factors affecting the resolution of $d l$-menthol by immobilized lipase-catalyzed esterification in organic solvent. J. Agric. Food Chem. 50, 262-265(2002).

6) Chen, Y.; Cheong, L.-Z.; Zhao, J.; Panpipat, W.; Wang, Z.; Li, Y.; Lu, C.; Zhou, J.; Su, X. Lipase-catalyzed selective enrichment of omega-3 polyunsaturated fatty acids in acylglycerols of cod liver and linseed oils: Modeling the binding affinity of lipases and fatty acids. Int. J. Biol. Macromol. 123, 261-268(2019).

7) Adlercreutz, P. Immobilisation and application of lipases in organic media. Chem. Soc. Rev. 42, 6406-6436 (2013).

8) Villeneuve, P.; Muderhwa, J.M.; Graille, J.; Haas, M.J. Customizing lipases for biocatalysis: A survey of chemical, physical and molecular biological approaches. $J$. Mol. Catal. B 9, 113-148(2000).

9) Brena, B.M.; Batista-Viera, F. Immobilization of enzymes. in Immobilization of enzymes and cells, Springer, pp. 15-30 (2006).

10) Basso, A.; Serban, S. Industrial applications of immobilized enzymes-A review. Mol. Catal. 479, 35-54 (2019).

11) Zou, B.; Hu, Y.; Jiang, L.; Jia, R.; Huang, H. Mesoporous material SBA-15 modified by amino acid ionic liquid to immobilize lipase via ionic bonding and cross-linking method. Ind. Eng. Chem. Res. 52, 2844-2851 (2013).

12) Yilmaz, E.; Can, K.; Sezgin, M.; Yilmaz, M. Immobilization of Candida rugosa lipase on glass beads for enantioselective hydrolysis of racemic Naproxen methyl ester. Bioresour. Technol. 102, 499-506 (2011).

13) Thangaraj, B.; Solomon, P.R. Immobilization of lipasesA review. Part I: Enzyme immobilization. ChemBioEng Reviews 6, 157-166 (2019).

14) Zhang, S.; Shi, J.; Deng, Q.; Zheng, M.; Wan, C.; Zheng, C.; Li, Y.; Huang, F. Preparation of carriers based on $\mathrm{ZnO}$ nanoparticles decorated on graphene oxide (GO) nanosheets for efficient immobilization of lipase from Candida rugosa. Molecules 22, 1205 (2017). 
15) Yan, M.; Ge, J.; Liu, Z.; Ouyang, P. Encapsulation of single enzyme in nanogel with enhanced biocatalytic activity and stability. J. Am. Chem. Soc. 128, 1100811009 (2006).

16) Li, Y.; Ruan, Z.; Zheng, M.; Deng, Q.; Zhang, S.; Zheng, C.; Tang, H.; Huang, F.; Shi, J. Candida rugosa lipase covalently immobilized on facilely-synthesized carbon nitride nanosheets as a novel biocatalyst. $R S C A d v$. 8, 14229-14236 (2018).

17) Zhang, S.; Deng, Q.; Li, Y.; Zheng, M.; Wan, C.; Zheng, C.; Tang, H.; Huang, F.; Shi, J. Novel amphiphilic polyvinylpyrrolidone functionalized silicone particles as carrier for low-cost lipase immobilization. $R$. Soc. Open Sci. 5, 172368(2018).

18) Zhang, S.; Deng, Q.; Shangguan, H.; Zheng, C.; Shi, J.; Huang, F.; Tang, B. Design and preparation of carbon nitride based amphiphilic Janus N-Doped Carbon/ MoS2 Nanosheets for Interfacial Enzyme Nanoreactor. ACS Appl. Mater. Interfaces 12227-12237 (2020).

19) Wang, H.; Zhang, H.; Wei, S.; Jia, Q. Preparation of ionic liquid hybrid melamine-based covalent organic polymer functionalized polymer monolithic material for the preconcentration of synthetic phenolic antioxidants. J. Chromatogr. A 1566, 23-31 (2018).

20) Yang, G.; Han, H.; Du, C.; Luo, Z.; Wang, Y. Facile synthesis of melamine-based porous polymer networks and their application for removal of aqueous mercury ions. Polymer 51, 6193-6202 (2010).

21) Sahiner, N.; Demirci, S.; Sel, K. Covalent organic framework based on melamine and dibromoalkanes for versatile use. J. Porous Mater. 23, 1025-1035(2016).
22) Jiang, C.; Cheng, C.; Hao, M.; Wang, H.; Wang, Z.; Shen, C.; Cheong, L.Z. Enhanced catalytic stability of lipase immobilized on oxidized and disulfide-rich eggshell membrane for esters hydrolysis and transesterification. Int. J. Biol. Macromol. 105, 1328-1336(2017).

23) Pencreac'h, G.; Baratti, J.C. Hydrolysis of $p$-nitrophenyl palmitate in $n$-heptane by the Pseudomonas cepacia lipase: A simple test for the determination of lipase activity in organic media. Enzyme Microb. Technol. 18, 417-422(1996).

24) Yun, T.; Yan, X. A modified para-nitrophenyl palmitate assay for lipase synthetic activity determination in organic solvent. Anal. Biochem. 363, 297-299 (2007).

25) Merline, D.J.; Vukusic, S.; Abdala, A.A. Melamine formaldehyde: Curing studies and reaction mechanism. Polym. J. 45, 413-419(2013).

26) Mircescu, N.E.; Oltean, M.; Chiş, V.; Leopold, N. FTIR, FT-Raman, SERS and DFT study on melamine. Vib. Spectrosc. 62, 165-171(2012).

27) Giacomini, C.; Villarino, A.; Franco-Fraguas, L.; Batista-Viera, F. Immobilization of $\beta$-galactosidase from Kluyveromyces lactis on silica and agarose: Comparison of different methods. J. Mol. Catal. B 4, 313-327 (1998).

28) Zhou, Q.Z.K.; Chen, X.D. Immobilization of $\beta$ galactosidase on graphite surface by glutaraldehyde. $J$. Food Eng. 48, 69-74(2001).

29) Spares, C.M.; De Castro, H.F.; De Morares, F.F.; Zanin, G.M. Characterization and utilization of Candida rugosa lipase immobilized on controlled pore silica. Appl. Biochem. Biotechnol. 77-79, 745-757 (1999). 